\title{
Photothermal beam deflection techniques: useful tools for integrated optics
}

\author{
H. EINSIEDEL, S. MitTLER-NehER
}

Max-Planck-Institut für Polymerforschung, Ackermann Weg 10, 55128 Mainz, Germany.

\begin{abstract}
Photothermal beam deflection spectroscopy (PTBDS) is used together with waveguide loss measurements to distinguish between intrinsic, absorptive and scattering losses in polymeric waveguide materials. Overtones of vibrational modes of organic waveguide materials in the near infrared spectral regime can be distinguished very precisely with PTBDS. In an example of different polythiophens, we demonstrate the influence of the side chains $\left(\mathrm{CH}_{2}\right.$ - and $\mathrm{CH}_{3}$-vibrations) and the conformation of the main chain on the absorption spectrum in the spectral regime of optical telecommunication. The photothermal beam deflection method is also used for imaging waveguide structures and to investigate order parameters of dyes within a polymeric matrix.
\end{abstract}

\section{Introduction}

All optical and electro-optical switching in waveguide geometries have attracted interest in the last years. Various classes of materials, for example, semiconductors glasses and polymers, have been investigated for these purposes [1]. Of these, polymeric materials are very attractive because they can be tailored by chemists to improve desired properties. Of course, high $\chi^{(3)}$ or $\chi^{(2)}$ values are desirable for optical or electro-optical switching at low input powers. It should also exhibit low linear losses and a relative high refractive index $n$ to assure a good field confinement in the waveguide [2].

Photothermal methods offer a wide variety of investigations on thermal and absorptive properties of matter without destruction [3], [4]. Especially the photothermal beam deflection technique or so-called mirage technique provides the possibility of detecting very weak absorptions in polymer films [5]. Inter alia, this was used to investigate the absorption spectra of vibrational overtones in the near infrared in homogeneous, isotropic polymer samples [6] and for thermal conductivity investigations [7]. Usually, the experimental set-ups work with a fixed sample and measure the properties in a spot thereof. Scanning photothermal beam deflection can image the lateral distribution of the absorption or the thermal conductivity within a sample. For these investigations the sample is slowly stepped in respect to the pump beam and the detected photothermal signal is plotted versus the scanning distance [8]. For the determination of order parameters of oriented chromophores within a polymer matrix a tunable polarizer can be used within the pump beam of the setup [9]. 
Polymeric waveguide materials should be processable so that waveguides can be fabricated. Processability for polymers often means solubility, which frequently is achieved at the cost of a reduced $\chi^{(3)}$ nonlinearity and a reduced refractive index [10], [11]. In the case of poly[3-alkylthiophen]s, the optically active part of the molecule is the conjugated main chain [12]. This main chain alone, without any side groups, is unsoluble and therefore not processable. To solve this problem side chains are attached to the main chain. In this case alkyl-side chains of different length had been introduced to the polythiophene main chain. To use these materials for a waveguide application one has to be sure not to hit an overtone of any possible vibrations of the side chains. In the near IR, the vibrational overtones are very weak but can be detected with photothermal techniques. We also investigated the influence on the absorption spectrum of the main chain conformation with respect to a head-to-head and a head-to-tail-coupling of the thiophene monomers forming the polymer.

We demonstrate the possibility of using poly (diazomethin-3-dodecyl thiophene) to fabricate channel waveguides and separate the linear losses into intrinsic absorptions and scattering losses. The optimal spectral region which is nearly free of absorption could be found [13].

As a sample material for the determination of order parameters we have chosen the dye DR1 in a matrix of PMMA, because this is a standard $\chi^{(2)}$ material [14], [15]. It is well known that the DR1 dye likes to undergo a trans-cis-trans isomerization cycle, which involves a conformation change, under excitation near its absorption maximum [15]. With this trans-cis-trans isomerization cycle the chromophore is oriented within the polymer matrix so that the dipole moment is aligned perpendicular to the polarization direction of the excitation beam. It is therefore very easy to align, not to pole DR1 molecules within a polymer matrix [16]. By orienting chromophores in such a way questions arise: How well are the chromophores oriented? Is there any damage by the laser power? Is the writing process reversible or irreversible?

\section{Experimental}

In the photothermal beam deflection microscopy and spectroscopy the incident pump beam is either a chopped $(8 \mathrm{~Hz})$ [17] cw frequency doubled Nd:YAG laser at $532 \mathrm{~nm}$ or a beam from a halogene lamp passed through a spectrometer. This beam is focused onto the sample surface. The pump beam is perpendicular to the sample surface and hits the surface on the front side. The sample is mounted in a cuvette, filled with a liquid (Fluorinert FC-72 ${ }^{\circledR}, 3 \mathrm{M}$ ) with a high $d n / d T$-coefficient to enhance the thermal lens in front of the laser focus. The sample cuvette can be moved in $x, y$-direction perpendicular to the pump beam by computer controlled translation stages. The detected signal beam from a $5 \mathrm{~mW}$ helium-neon laser (at $\lambda=632.8 \mathrm{~nm}$ ) propagating parallel to the sample surface is tightly focused down in front of the sample exactly where the pump beam focus is located. Due to the thermal lens in front of the sample which is formed through the absorption of the pump beam in the 


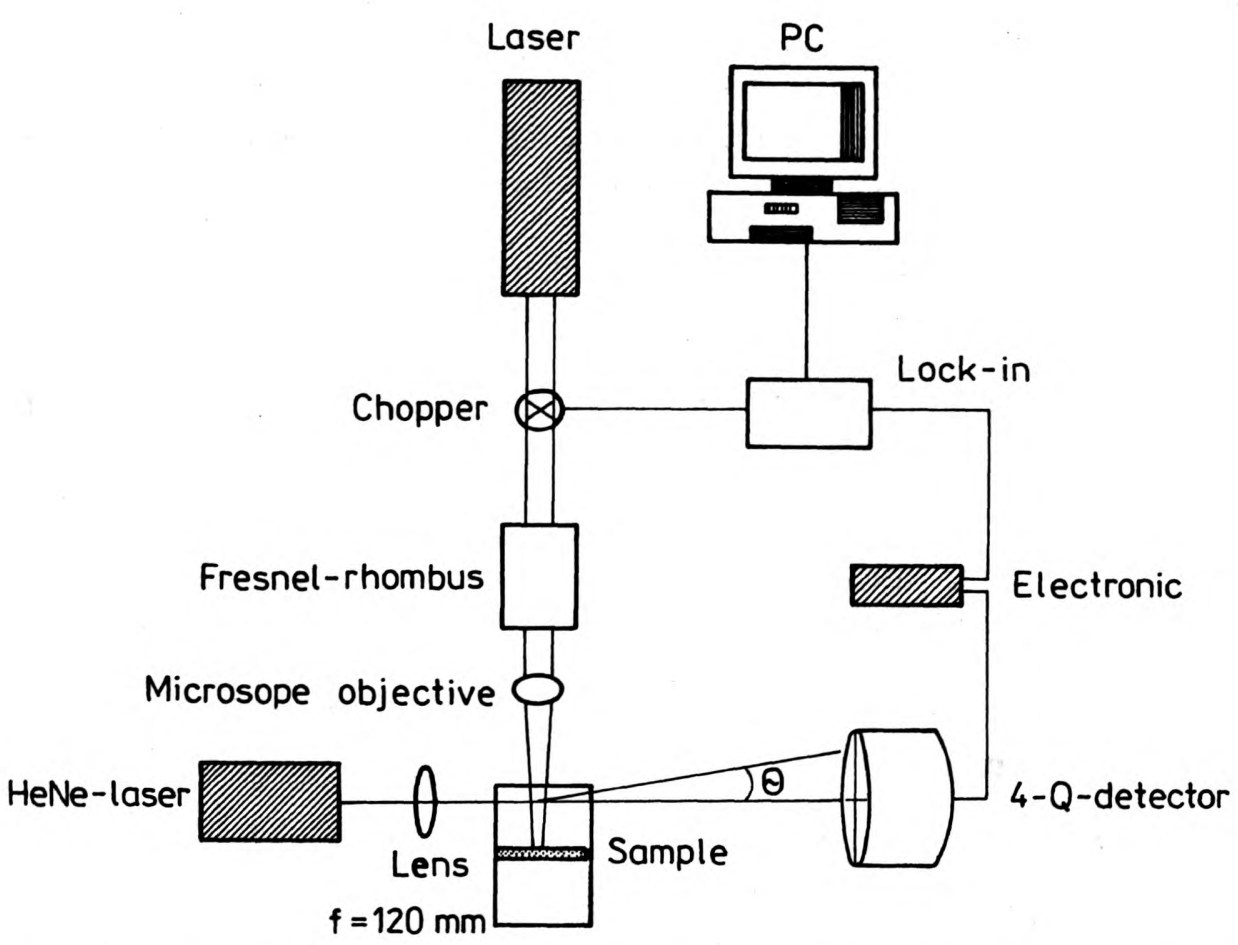

Fig. 1. Scheme of the experimental setup for the photothermal beam deflection microscopy

sample, the He-Ne laser beam is deflected away from the sample surface. The deflected beam is detected by a position sensitive detector (UDT) and the signal processed by a PC. In the case of the order parameter determination a Fresnel-romb was added in the laser pump beam. This experimental setup is depicted in Fig. 1.

For the determination of the waveguide losses, a standard waveguide characterisation setup was used [11], [18]. The polarized laser beam was coupled into a slab waveguide $(4.2 \mu \mathrm{m})$ by a grating, which was fabricated holographically and ion milled into the fused silica substrate [19]. The image of the streak, taken with an IR sensitive video camera (Electrophysics) has been digitalized by a commercial software (Image-Pro Plus). The resulting intensity profile was fitted to a decreasing exponential function and the loss in $\mathrm{dB} / \mathrm{cm}$ was calculated.

\section{Sample preparation}

In this study we used poly[4-butylthiophene] (PT4), poly[3-hexylthiophene] (PT6), poly [3-decylthiophene] (PT10), poly [3-tetradecylthiophene] (PT14) and poly[diazomethin-3-dodecylthiophene] (PT12). PT6 had been available in a head-to-head- and head-to-tail-monomer coupling configuration. The synthesis and the preparation of the poly[3-alkylthiophene]s are described previously [13], [20]. The polymers were dissolved in chloroform (Merck, pA-quality) and shortly put into an ultrasonic bath 
at $30^{\circ} \mathrm{C}$. The concentration ranged from 2 to $10 \% \mathrm{w} / \mathrm{v}$. The solution was then cast onto a glass substrate and dried 60 minutes at $50^{\circ} \mathrm{C}$ in a vacuum oven for solvent evaporation to form films of typical thicknesses of $30 \mu \mathrm{m}$.

For the waveguide fabrication a $850 \mathrm{~nm}$ thick PT12 film was spincoated on top of a fused silica slide. The losses had been measured with this slab waveguide sample. For the channel waveguide formation, the sample was then placed in a contact mask aligner (Cobilt) and irradiated $72 \mathrm{~h}$. The coupling into the channel waveguide structures was done by a prism. This PT12 is not too soft for doing so. The prism could be removed without damaging the film.

The dye containing polymer films has been spincoated at 3000 RPM out of a $10 \%$ of weight DR1 in PMMA (polymethylmetacrylate) as a guest-host system in ethoxymethylacetat solution. The resulting film thickness after $24 \mathrm{~h}$ of drying at room temperature was $1.8 \mu \mathrm{m}$. To orient the chromophores in the sample the linear polarized, frequency doubled $\mathrm{cw}$ Nd:YAG laser $(532 \mathrm{~nm})$, which is also used as the pump source in the photothermal beam deflection experiment, was focused onto the sample.

\section{Results and discussion}

\subsection{Poly[diazomethin-3-dodecylthiophene] (PT12)}

In Figure 2, thePBD spectrum of PT12 is shown. The increasing absorption on the small wavelength side is due to the electronic absorption in the visible of the conjugated main chain [11]. In the near IR clearly a variety of vibrational overtones can be found. They are due to the alkyl side chains. The absorption bands marked with arrows are due to pure overtones of stretch-vibration of the order $x(x, 0)$ and combination bands of stretch- $(x)$ - and bend- $(y)$-vibration $(x, y)$ of the $\mathrm{CH}_{3} / \mathrm{CH}_{2}$-group. The aromatic $\mathrm{CH}$-stretch vibration is too weak to be detected as overtones.

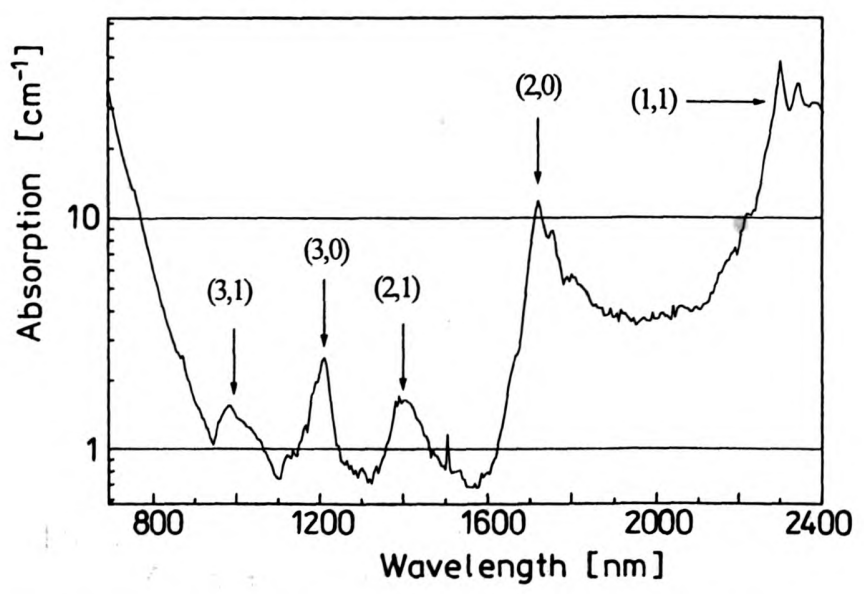

Fig. 2. Photothermal beam deflection spectrum of PT12 
The absorption coefficient for $1064 \mathrm{~nm}$ is $4.3 \mathrm{~dB} / \mathrm{cm}$ and for $1150 \mathrm{~nm}-4.6$ $\mathrm{dB} / \mathrm{cm}$. The losses measured with the $\mathrm{TE}_{0}$ modes of the unbleached slab waveguide are $11.3 \mathrm{~dB} / \mathrm{cm}$ for $1064 \mathrm{~nm}$ and $10.2 \mathrm{~dB} / \mathrm{cm}$ for $1150 \mathrm{~nm}$. It is found for both wavelengths that more than $50 \%$ of the loss is due to scattering. For higher waveguide modes (up to $\mathrm{TE}_{4}$ and $\mathrm{TM}_{4}$ ) the losses increase up to $15 \mathrm{~dB} / \mathrm{cm}$ - to the dominant loss feature - which indicates that surface scattering is an important problem. The best spectral region, concerning the vibrational overtones and electronic absorptions, for using this material as a waveguide is at $1550 \mathrm{~nm}$, one of the communication laser wavelengths, with an intrinsic absorption coefficient of $3.0 \mathrm{~dB} / \mathrm{cm}$, which is still too high for nonlinear optical applications. Here is the lowest gap between two absorption bands of overtones.

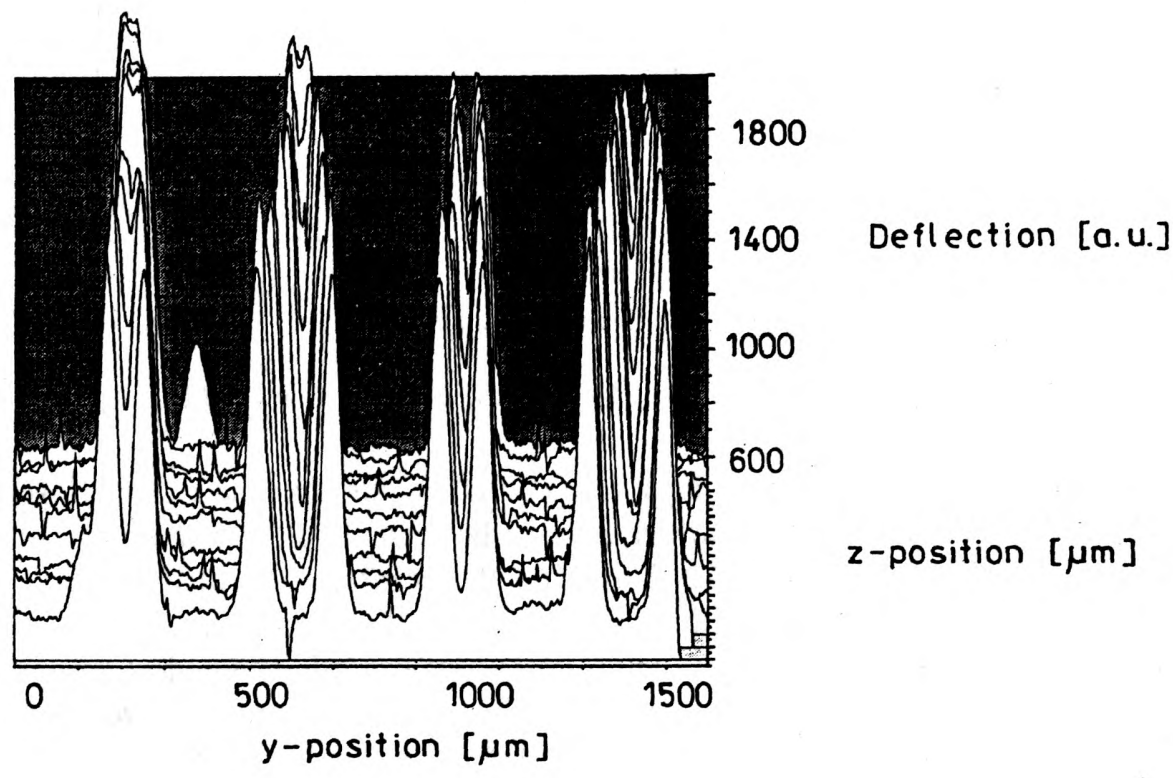

Fig. 3. Photothermal image of PT12 channel waveguides. Four $y$-junctions can be seen

The absorption coefficient difference, which occurs in parallel to the refractive index change during bleaching, is depicted in a 3D plot in the PBD-image of the channel waveguides in Fig. 3. The high stripes are the unbleached channel waveguides and the deep valleys are the bleached areas. Four $y$-junctions are depicted here. The sharp slopes of the channel waveguide margins show the good quality of the fabrication process.

\subsection{Poly[alkylthiophenes]}

Figure 4 shows normalized PBD spectra of PT4, PT6, PT10 and PT14. In the PT4 spectrum only a weak shoulder on top of the background absorption can be found where the $v_{s}^{2}(\mathrm{C}-\mathrm{H})$ and $v_{a}^{2}(\mathrm{C}-\mathrm{H})$ bands of the $\mathrm{CH}_{2}$ groups are located. The PT6 is the 


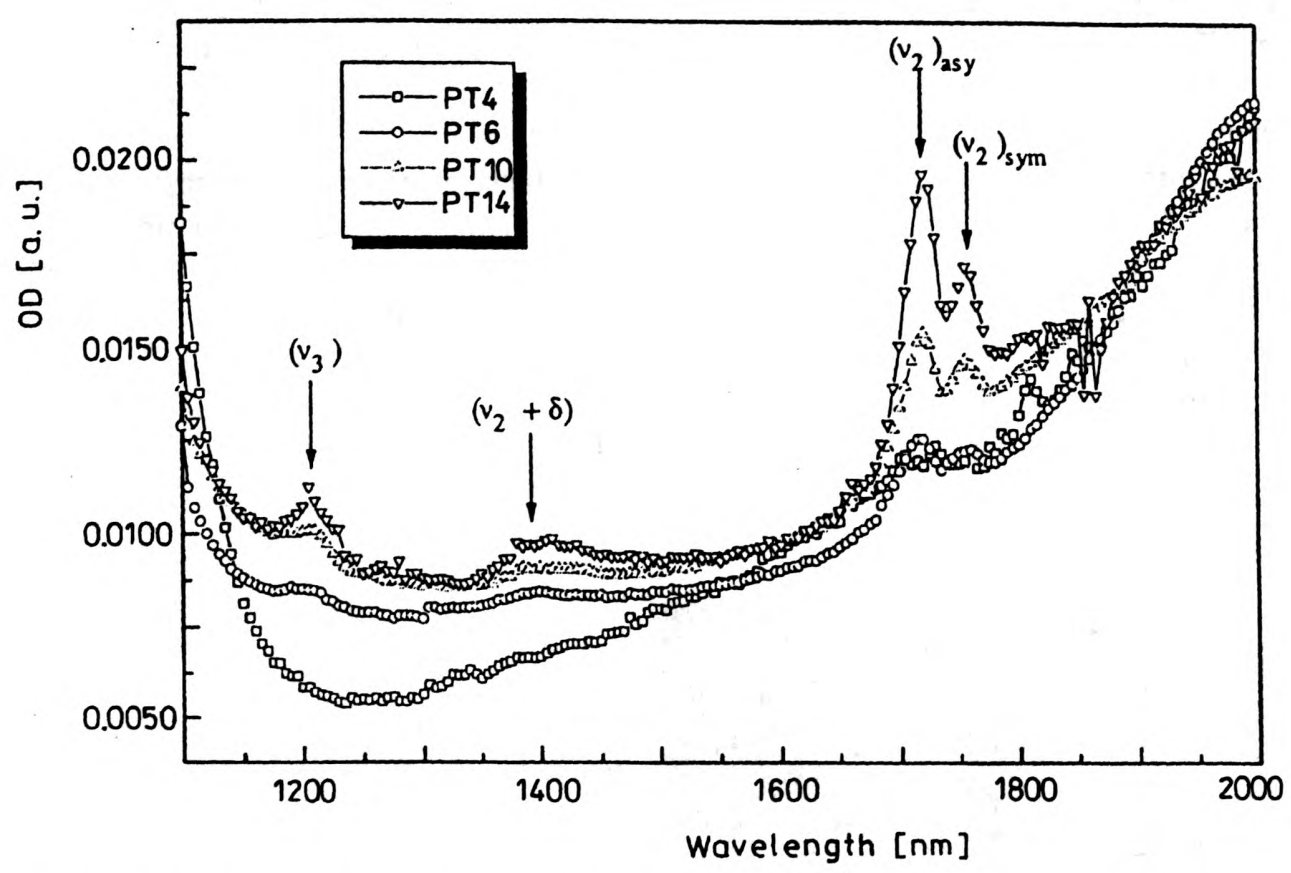

Fig. 4. Normalized photothermal beam deflection spectra of PT4, PT6, PT10 and PT14

first compound which shows the two distinct vibrational bands. An increase of both peak intensities with increasing side chain length can be seen. The third overtone of the $\mathrm{CH}_{2}$ vibrations $v^{3}(\mathrm{C}-\mathrm{H})$ at around $1200 \mathrm{~nm}$ and the combination of the stretch vibration with the second overtone of the $\mathrm{CH}_{2}$ vibrations $\left(v^{2}+\delta\right)(\mathrm{C}-\mathrm{H})$ at around $1400 \mathrm{~nm}$ can be found only clearly in the spectra of the longer side chain derivatives PT10 and PT14. The peak intensities of the $v_{s}^{2}(\mathrm{C}-\mathrm{H})$ and $v_{a}^{2}(\mathrm{C}-\mathrm{H})$ bands have been normalized by the number of $\mathrm{CH}_{2}$-groups in the side chain. For both bands the increase in intensity is overlinear with increasing side chain. The absorbance per $\mathrm{CH}_{2}$-group increases from 0.34 to 0.55 for the asymmetric and from 0.14 to 0.28 for the symmetric vibration from PT6 to PT14. This can cause serious loss problems in nonlinear optical devices due to one and two photon absorption when the side chain length is increased. This increase is necessary because the PT14 has still a too short side chain in order to spincoat waveguides with low scattering losses.

All PT spectra show a very clear shoulder around $1000 \mathrm{~nm}$ in the long wavelength decay of the main absoption. To our knowledge this shoulder has not been reported in absorption spectra so far for PTs. In Figure 5 the spectra for the two PT6 species, head-head-head-to-tail and a head-to-tail coupled, show a distinct difference. The species with the head-head-head-to-tail coupling does not show the absorption band at $970 \mathrm{~nm}$. This additional band cannot be attributed to vibrational overtones of the side chains. So it must be attributed to the main chain. The difference in the main chain is the delocalization length of the $\pi$-electron. A head-to- 


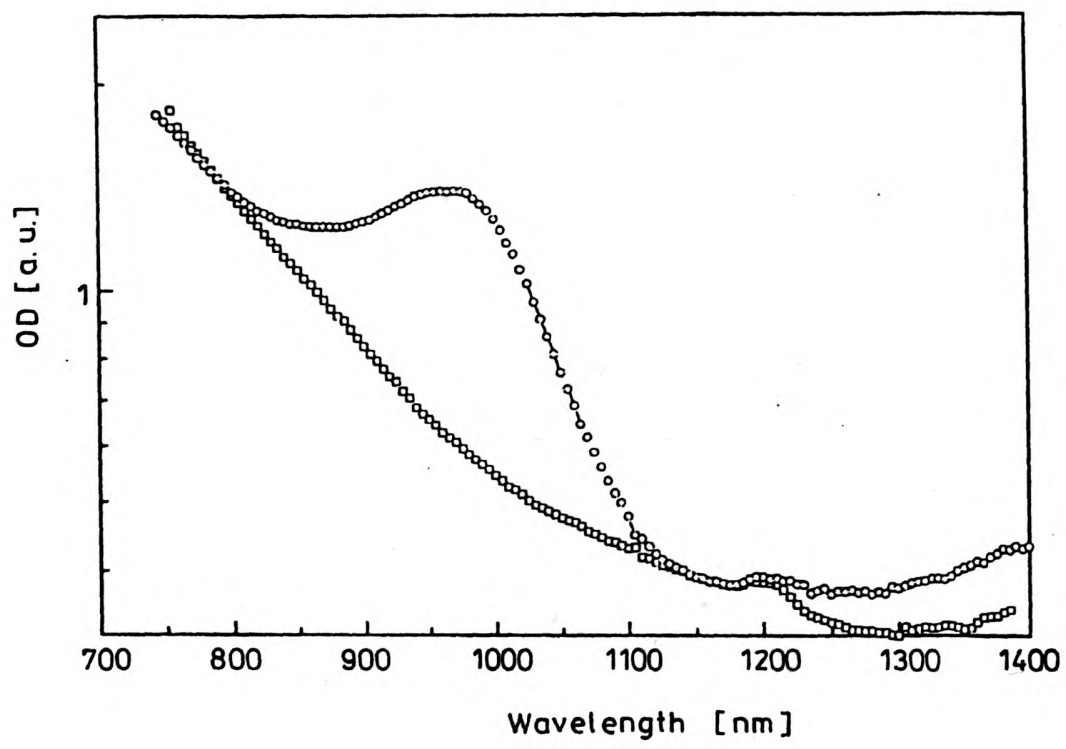

Fig. 5. Photothermal beam deflection spectra of the two species of PT6: 0 - head-to-tail/head-to-head, $\square$ - head-to-tail

head coupling results in a steric hinderance of the alkyl chains which leads to a twist of the bond between two neighbouring thiophene rings. This disturbes the conjugation of the system [20]-[24].

A similar behaviour was also found by measuring the $\chi^{(3)}$-value for neutral poly[3-decylthiophen]s, which are strongly dependent on the length of the conjugated system. The PT with the highest degree of head-to-tail-coupling showed the highest nonlinear response [25]. VARDENY et al. [26] and SATO et al. [27] have proposed bipolaron states in the gap in a spectral regime of $990 \mathrm{~nm}$ and $2700 \mathrm{~nm}$ for pure neutral PT and slightly shifted for doped derivatives. Vardeny et al. used neutral PT and conclude that the bipolarons are formed by a photoinduced process. Sato et al. find a bipolaron after doping. The intensity ratio of the two transitions in the neutral PT measured by Vardeny et al. is 1.5 . We find a similar ratio in our neutral PT6 between the peak at 970 and $2380 \mathrm{~nm}$. Because we do not photo-induce the bipolarons with our PTB deflection technique, the material has to be slightly doped. This might be due to residual iron from the synthesis [20]. These dopants can in principle be responsible for the formation of a charged excitation.

\subsection{DR1/PMMA}

An isotropic PMMA/DR1-guest-host sample has been investigated with a pump power density of $150 \mathrm{~W} / \mathrm{cm}^{2}$. The deflection signal was measured while turning the polarization direction $\varphi$ of the pump beam. The result is depicted in Fig. 6 in the form of a polar diagram. There are no oscillatory features in the outer most circle (open triangles) of the diagram. However, there is a clear gap at $360^{\circ}$ due to 


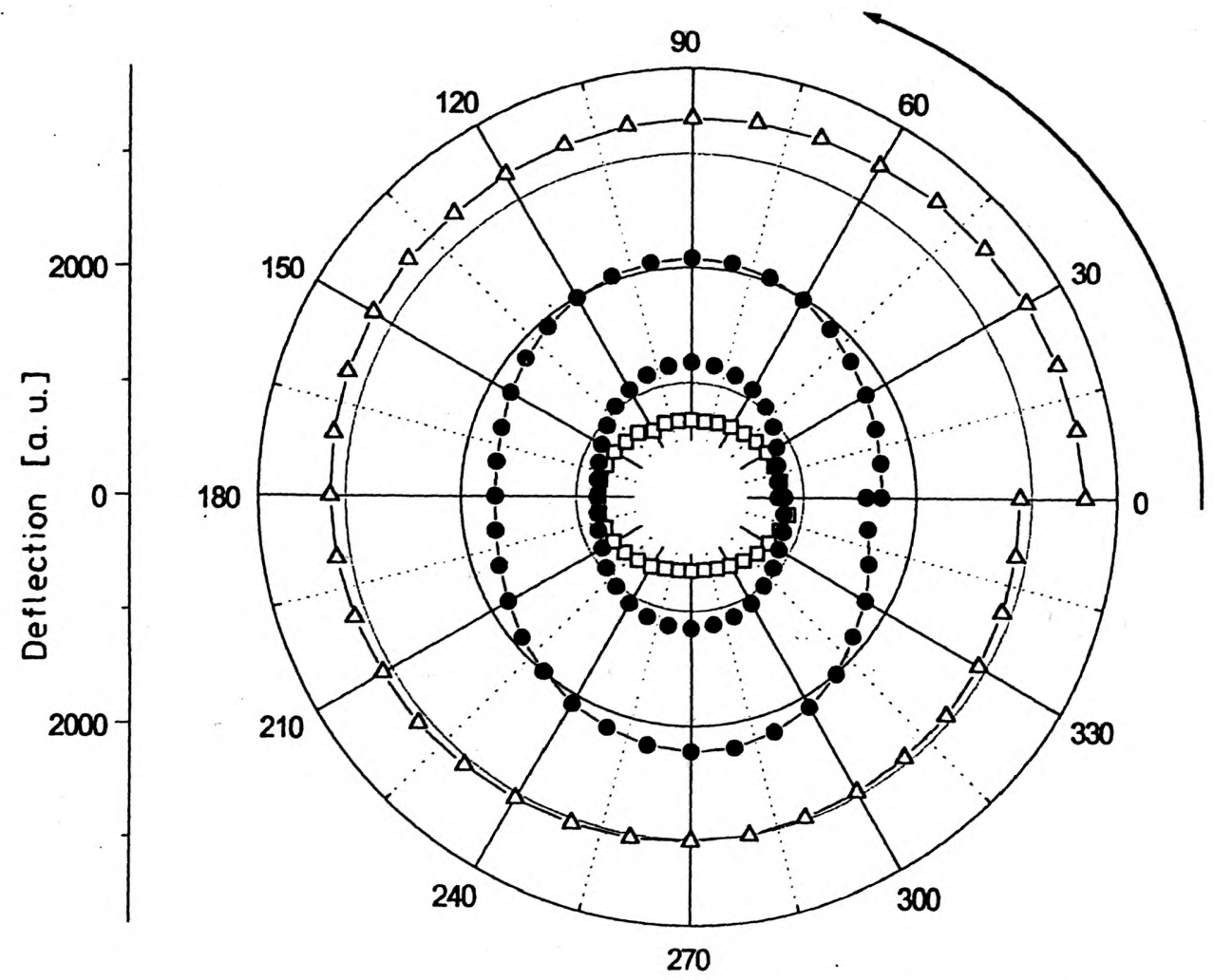

Fig. 6. Photothermal beam deflection signals in form of a polar diagram of DR1/PMMA guest-host system as unbleached $(\Delta)$, shortly bleached (outer signal $\bullet$ ) and longer bleached (inner signal $\bullet$ ) with a polarization parallel to the $0-180^{\circ}$ axis and of an additional bleaching with a polarization direction parallel to the $90-270^{\circ}$ axis (口)

irreversible dye bleaching. After illuminating the sample with a power of $30 \mathrm{~kW} / \mathrm{cm}^{2}$ with a polarization parallel to the axis $0-180^{\circ}$ for several minutes, an oscillating behaviour of the deflection signal with respect to the polarization direction $\varphi$ of the absorbed beam could be seen clearly. The circle was dented in form of a cigar with the long axis perpendicular to the polarization direction of the bleaching laser beam. The absorption is therefore larger perpendicular to the polarization direction of the bleaching laser beam than parallel to it. This is due to the orientation of the dipole moments within the plane of the sample away from the bleaching polarization direction. A high deflection corresponds to a high absorption, because of a constructive overlap of the dipole moment of the chromophore with the polarization direction of the bleaching beam. By increasing the time of bleaching the deflection signal decreases in general and the cigar shape in the polar diagram is "stable". By changing the polarization direction of the bleaching beam parallel to the $90-270^{\circ}$ line the deflection signal decreases again and the long axis of the cigar is turned by $90^{\circ}$ as well (open squares), again perpendicular to the polarization direction of the bleaching beam. 
The data from the polar diagram can also be plotted in the form of deflection versus angle. This results in a sinusoidal square curve which can be fitted to

$$
A=A_{\perp} \sin ^{2} \varphi+A_{1} \cos ^{2} \varphi,
$$

with $A$ - the deflection signal, $A_{\perp}-$ a fit parameter representing the absorption component perpendicular to the polarization, $A_{\mathbf{l}}-$ a fit parameter representing the absorption component parallel to the polarization and $\varphi$ - the polarization angle. With

$$
C_{2}=\frac{A_{\perp}+A_{\mathbf{l}}}{2 A_{\perp}+A_{\mathbf{l}}}
$$

the order parameter $C_{2}$ can be calculated [28].

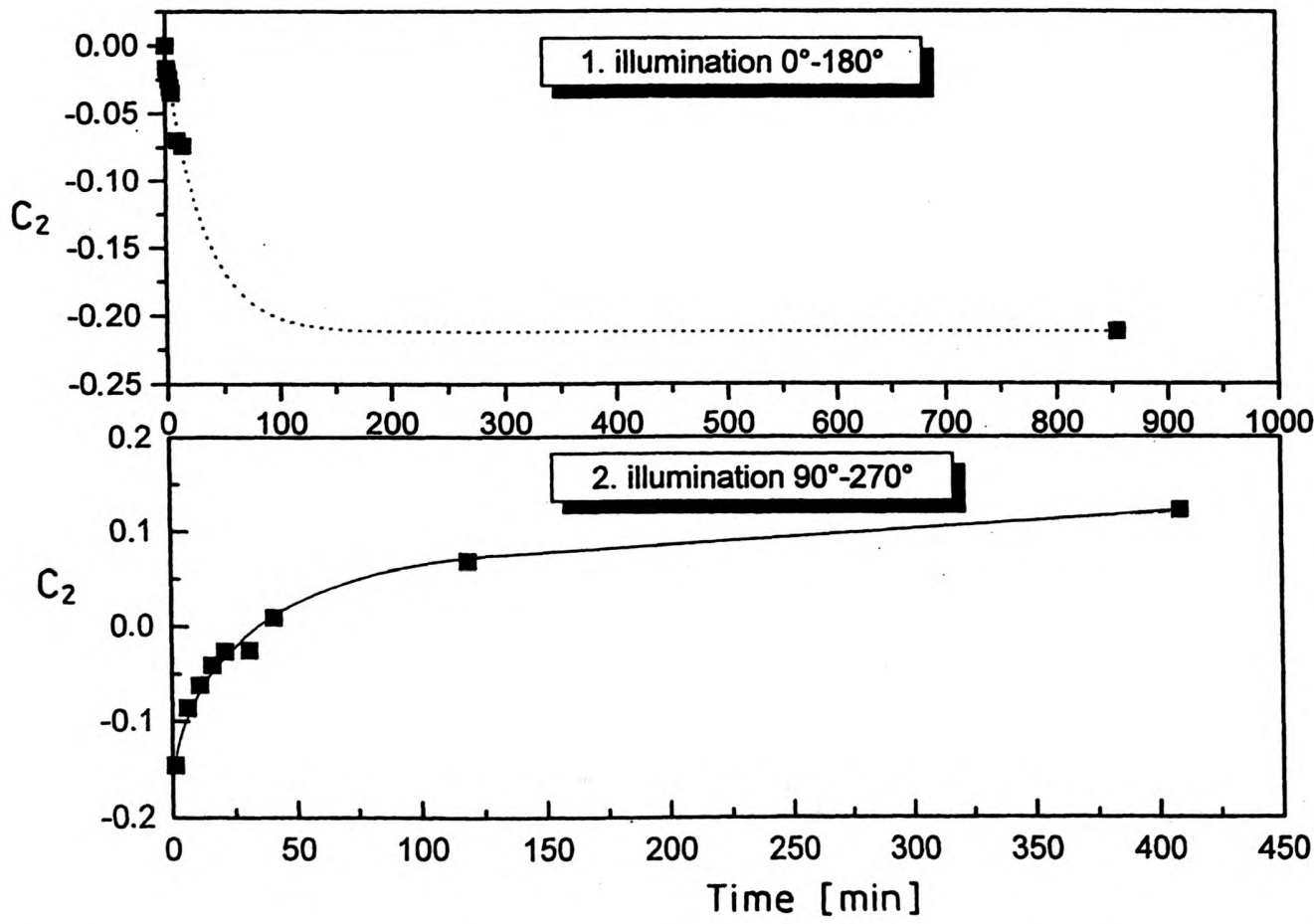

Fig. 7. Order parameter time development with orientation and reorientation of the dye by pumping with a polarization direction parallel to the $0-180^{\circ}$ axis in the: 1. illumination (upper graph) and afterwards with a polarization parallel to the $90-270^{\circ}$ axis in the 2. illumination (lower graph)

We have performed a reorientation experiment. First the DR1/PMMA sample had been oriented with a polarization direction parallel to the axis $90-270^{\circ}$ and after this again, but with perpendicular polarization direction $\left(0-180^{\circ}\right)$. The orientation cigar is turned by an angle of $90^{\circ}$ by switching the direction of the polarization of the pump beam. The time behaviour of the orientation process 
for both polarization directions is shown in Fig. 7. The order parameter increases exponentially and reaches an end value. By starting the reorientation with the perpendicular polarization the order parameter first decreases to 0 and then increases to nearly the same value of the order parameter as before. It is definitely possible to switch the molecules gently without severe damage between two molecular orientations.

\section{Conclusions}

Photothermal beam deflection spectroscopy and microscopy in combination with waveguide loss investigation is a helpful set of tools for integrated optics. Waveguide inspection, identification of loss mechanisms, finding of wavelength windows for the application, the determination of order parameters of oriented chromophores, and so on, can be achieved by these tools.

Acknowledgements - We like to thank Mario Leclerc for providing the poly[alkylthiophens] and Claudio Amari for the synthesis of the poly[diazomethin-3-dodecylthiophene]. We also like to acknowledge all the help of Bernhard Menges on the waveguide fabrication and characterization.

\section{References}

[1] Stegeman G. I., Seaton C. T., Zanoni R, Thin Solid Films 152 (1987), 231.

[2] KARTHE W, MÚlleR R., Integrierte Optik, Akademische Verlagsgesellschaft Geest\&Portig KG, Leipzig 1991.

[3] Biancic D, Photoacoustic and Photothermal Phenomena III, Springer Series in Optical Sciences, Bd. 69, Heidelberg 1992.

[4] Proceedings of the 8th International Topical Meeting on Photoacoustic and Thermal Phenomena, J. Phys. IV, 4, Suppl. III (1994).

[5] Jackson W. B., Amer N. M, Boccara A. C., Fourier D, Appl. Opt. 20 (1981), 1333.

[6] Seager C. H., Sinclatr M., McBranch D, Heeger A. J., Baker G. L., Synth. Metals 49-50 (1992), 91.

[7] Rantala J., Wei L., Kuo P. K, JaArinen J., LuUkKalam M., Thomas R L. , J. Appl. Phys. 73 (1993), 2714.

[8] Einsiedel H., Mittler-Neher S, Thin Solid Films, in press.

[9] Einsiedel H., Mittler-Neher S., Appl. Opt., in press.

[10] Torruellas W. E., Neher D., Zanoni R., Stegeman G. I., Kajzar F., Leclerc M., Chem. Phys. Lett. 175 (1990), 11.

[11] Lee T.-M., Mittler-Neher S., Neher D., Stegeman G. I., Roux C., Leclerc M., Opt. Materials 1 (1992), 65.

[12] Tienpot H., Rikken G. L. J. A., Meijer E. W., Ten Hoeven W., Wynberg H., Phys. Rev. Lett. 65 (1990), 2141.

[13] Amari C, Pelizzi C, Predieri G., Destri S., Porzio W., Einsiedel H., Mittler-Neher S., J. Mat. Chem., in press.

[14] Sexkat Z, AUSt E. F., Knoll W., [In] Reprints from ACS Symposium Series, No. 601, Chapt. 19 (1995), p. 255.

[15] Dumont M., Hosotte S., Froc C., SekKat Z., Proc. SPIE 2042 (1993), 1.

[16] SekKat Z, Dumont M., Synth. Metals 54 (1993), 378.

[17] Bain C. D, Davies P. B, ONG T. H., Chem. Phys. Lett. 194 (1992), 391.

[18] TAMIR T., Integrated Optics, Topics in Applied Physics, Vol. 72, 2nd edition, Springer, Berlin 1979. 
[19] Mai X., Moshrafzadeh R., Gibson U. J., Stegeman G. I., Seaton C. T., Appl. Opt. 24 (1985), 3155. [20] Leclerc M, Diaz F. M, Wegner G., Makrom. Chem. 190 (1989), 3105.

[21] Robitaille L., LecleRC M., Macromolecules 27 (1994), 1847.

[22] Souto Maior R. M., Hinkelmann K., ECKert H., WUdl F., Macromolecules 23 (1990), 1268.

[23] Mc Cullough R. D., Lowe R. D, Jayaraman M., Anderson L. D, J. Org. Chem. 58 (1993), 904.

[24] CheN T., Wu X., RIEKe R. D., JACS 117 (1995), 233.

[25] Neher D., Wolf A., Leclerc M., Kaltbeitzel A., Bubeck C., Wegner G, Synth. Metals 37 (1990), 249.

[26] Vardeny Z., Ehrenfreund E., Brafman O., Novak M., Schaffer H., Heeger A. J, Wudl F., Phys. Rev. Lett. 56 (1986), 671.

[27] Sato M., Tanaka S., Kaeriyama K, Synth. Metals 14 (1986), 279.

[28] Heher D., Mittler-Neher S., Cha M., Stegeman G. I., Embs F. W., Wegner G., Miller R. D., WILson C. G., Proc. SPIE 1560 (1991), 335. 